\title{
Suppression of grasshopper sound production by nitric oxide-releasing neurons of the central complex
}

\author{
Anja Weinrich • Michael Kunst • Andrea Wirmer • \\ Gay R. Holstein $\cdot$ Ralf Heinrich
}

Received: 25 March 2008 / Revised: 27 May 2008 / Accepted: 28 May 2008 / Published online: 24 June 2008

(C) The Author(s) 2008

\begin{abstract}
The central complex of acridid grasshoppers integrates sensory information pertinent to reproductionrelated acoustic communication. Activation of nitric oxide (NO)/cyclic GMP-signaling by injection of NO donors into the central complex of restrained Chorthippus biguttulus females suppresses muscarine-stimulated sound production. In contrast, sound production is released by aminoguanidine (AG)-mediated inhibition of nitric oxide synthase (NOS) in the central body, suggesting a basal release of NO that suppresses singing in this situation. Using anti-citrulline immunocytochemistry to detect recent NO production, subtypes of columnar neurons with somata located in the pars intercerebralis and tangential neurons with somata in the ventro-median protocerebrum were distinctly labeled. Their arborizations in the central body upper division overlap with expression patterns for NOS and with the site of injection where NO donors suppress sound production. Systemic application of AG increases the responsiveness of unrestrained females to male calling songs. Identical treatment with the NOS inhibitor that increased male song-stimulated sound production in females induced a marked reduction of citrulline accumulation in central complex columnar and tangential neurons. We conclude that behavioral situations that are unfavorable for sound production (like being restrained) activate NOS-expressing central
\end{abstract}

\footnotetext{
A. Weinrich · M. Kunst · A. Wirmer · R. Heinrich ( $\square)$ Department of Neurobiology, Institute of Zoology, University of Göttingen, Berliner Strasse 28, 37073 Göttingen, Germany e-mail: rheinri1@gwdg.de

G. R. Holstein

Mount Sinai School of Medicine,

City University of New York, New York, NY, USA
}

body neurons to release NO and elevate the behavioral threshold for sound production in female grasshoppers.

Keywords Nitric oxide - Central complex · Behavior . Sound production - Grasshopper
List of abbreviations
AG Aminoguanidine hemisulfate
cGMP Cyclic guanosine monophosphate
mAChR Muscarinic acetylcholine receptor
NADPH Reduced form of nicotinamide adenine
dinucleotide phosphate
NO Nitric oxide
NOS Nitric oxide synthase
PB Phosphate buffer
sGC Soluble guanylate cyclase
SNP Sodium nitroprusside

\section{Introduction}

Nitric oxide (NO) has been established as an important signalling molecule in nervous systems of both vertebrates (Garthwaite and Boulton 1995; Prast and Philippu 2001) and invertebrates including insects (reviews: Colasanti and Venturini 1998; Davies 2000; Bicker 2001; Moroz 2001; Heinrich and Ganter 2007). In nervous tissues, gaseous NO is produced on demand by $\mathrm{Ca}^{2+} /$ calmodulin-activated nitric oxide synthase (NOS) and diffuses freely across cell membranes to stimulate the production of cGMP primarily through its target receptor soluble guanylyl cyclase (sGC). Genes coding for NOS and sGC have been identified in a number of insect species and their expression and function has been demonstrated in various types of glia, receptor neurons, interneurons, motoneurons and neurosecretory 
cells (reviews: Bicker 2001; Trimmer et al. 2004). Recent studies have demonstrated the prominent presence of NOS in almost all central nervous system structures (Ott and Elphick 2002, 2003; Kurylas et al. 2005), suggesting that the impact of NO signalling on neural information processing may be significantly underestimated.

Studies on a variety of insects indicate that NO-initiated signalling pathways participate in the development, structural organization and maintenance of central nervous neuropils, as well as in the control of insect behaviors on various functional levels including perception and processing of sensory information, its integration with an individual's internal state and adaptive modulation of neuro-muscular and neurosecretory systems (reviewed by: Müller 1997; Bicker 1998, 2001; Trimmer et al. 2004; Heinrich and Ganter 2007). In addition, NO-stimulated formation of cGMP can regulate thresholds for particular behaviors through modulation of neural excitability and synaptic transmission properties in central nervous regions concerned with the selection and coordination of those behaviors. Prominent examples of this include foraging in Drosophila larvae stimulated under hypoxic conditions (Osborne et al. 1997; Wingrove and O'Farrell 1999), female pheromone-induced searching behavior of male Bombyx mori (Seki et al. 2005), experience-dependent fighting in crickets (Aonuma et al. 2004) and grasshopper sound production controlled by the central body (Wenzel et al. 2005), the subject of the present study.

Nitric oxide synthase and NO-stimulated accumulation of cGMP have been detected in the central complex of various insects (reviewed by Müller 1997). The central complex represents a midline-spanning assembly of four interconnected neuropils: the protocerebral bridge, the upper and lower divisions of the central body and the noduli. The central body receives multimodal sensory information pre-processed by other central nervous system regions. It has been demonstrated to be involved in spatiotemporal sensory processing, selection of behaviors, motor coordination and spatial orientation (Bausenwein et al. 1994; Ilius et al. 1994; Heinrich et al. 2001; Strauss 2002; Heinze and Homberg 2007; reviewed by Wessnitzer and Webb 2006). In grasshoppers (detailed references listed below) and fruitflies (Popov et al. 2005), the central body functions as the major control center for the selection and coordination of sound production in the context of matefinding, courtship and rivalry (Elsner 1974; Elsner and Popov 1978). Pharmacological studies on restrained but intact grasshoppers of various species have identified multiple signalling pathways including ionotropic and metabotropic receptors within the central body neuropils that promote (acetylcholine: Heinrich et al. 1997, proctoline and dopamine: unpublished results) or inhibit (GABA, glycine: Heinrich et al. 1998, NO: Wenzel et al. 2005) sound production. This has led to the idea that the central complex integrates external and internal sensory information representing an individual's immediate environment and physiological state in order to modulate the thresholds for both activation of the most appropriate behavior for the situation and suppression of inappropriate behaviors (Heinrich et al. 2001; Hoffmann et al. 2007). Injections of NO donors, membrane permeable analogs of cGMP and inhibitors of cGMP-dependent phosphodiesterase into the central body have all been shown to suppress muscarine-stimulated sound production and this suppression disappeared when sGC was inhibited (Wenzel et al. 2005). Histological and immunocytochemical studies detected elaborate networks of NOS-containing neurites in the upper division of the central body and NO-stimulated accumulation of cGMP in the lower division (Wenzel et al. 2005).

In the present study, we have extended our previous studies on restrained grasshoppers to systemic drug applications combined with behavioral experiments and chose female grasshoppers as experimental subjects. Spontaneous sound production in females depends on the reproductive state and is strictly correlated with high receptivity after prolonged periods without copulation (Loher and Huber 1964). We demonstrate here that the release of NO in the central body suppresses sound production and that inhibition of NOS in the central body of restrained females is sufficient to release sound production, suggesting that $\mathrm{NO}$ is tonically released in this neuropil under conditions of restraint. Using anti-citrulline immunocytochemistry, we identified columnar and tangential neurons in the central body that actively release NO and may therefore be responsible for the suppression of sound production in this inhospitable situation. Injections of the NOS inhibitor aminoguanidine (AG) into the hemolymph of female grasshoppers reduced accumulation of citrulline in central body neurons and an enhanced responsiveness to stimulation with male calling songs. We conclude from these results that behavioral situations that are unfavorable for sound production (such as being restrained) activate NOSexpressing central body neurons whose release of NO modulates synaptic processes resulting in the elevation of the behavioral threshold for sound production in grasshoppers.

\section{Materials and methods}

Animals

Experiments were performed with adult female grasshoppers of the species Chorthippus biguttulus (L.) caught in the vicinity of Göttingen (Germany). Virgin females were reared from eggs that were collected during the previous summer and kept at $4{ }^{\circ} \mathrm{C}$ for at least 4 months. After molting 
into the 4th nymphal instar, females were selected and kept in a separate room to avoid both mating and acoustic stimulation by males. Experiments with virgin females started 5-10 days after their imaginal molt.

Pharmacological stimulation of sound production in restrained female grasshoppers

Grasshoppers were restrained with wax, the head capsules opened from the dorsal side of the body, and the brains tipped forward to expose their dorsal (with respect to the neural axis) surfaces. Solutions of muscarine, sodium nitroprusside (SNP), AG hemisulfate salt (all from Sigma), S-methylL-thiocitrulline (Cayman Chemical) and $\mathrm{N}^{G}$-nitro-L-arginine (Alexis Biochemicals) were dissolved in grasshopper saline (Clements and May 1974). Neuroactive drugs or physiological saline (control) were applied through single or double barreled capillaries coupled to a pressure pump (WPI model PV 820), enabling injections of approximately 1-3 nl into targeted brain neuropils. This was confirmed by injections from the same capillaries that were used for brain stimulation into paraffin oil, measuring the size of the spherical droplets under a microscope and calculating their volume. Prior to testing for potential inhibitory effects of SNP on muscarine-stimulated stridulation, the average duration of muscarine-stimulated songs was calculated from three consecutive trials. Identical muscarine injections were applied in regular intervals of $5 \mathrm{~min}$ throughout the entire experimental series to maintain a similar level of overall excitation. The test substance was injected into the same site within the brain, between two regular stimulations with muscarine. For comparison of duration and latency of muscarine- and NOS inhibitor-stimulated stridulation, the two drugs were injected to the same site within the grasshopper brain. In order to avoid long lasting interfering effects from the previous stimulation, injection intervals of 15 min were maintained and the drug applied as the initial stimulus was varied across experiments. Stridulatory hind leg movements were recorded with optoelectronic cameras (von Helversen and Elsner 1977) and, together with recordings of sound and the injection pulse, digitized and stored on a personal computer. Data were processed with the analysis program NEUROLAB (Hedwig and Knepper 1992) and figures were generated with PHOTOSHOP (Version 9, Adobe Systems).

Pharmacological studies with acoustically stimulated unrestrained females

Adult female grasshoppers isolated from males since their 4th nymphal instar were kept at a regular light/dark cycle of 12:12 h. During experimental series, animals were injected every morning at the same time with $30 \mu \mathrm{l}$ of either $10^{-2} \mathrm{M}$ AG diluted in saline or saline alone as control. Injections were applied into the abdominal hemolymph while the grasshoppers were immobilized on a chilled metal holder. After injections, each female was maintained in a small individual cage that contained wheat and substrate for egg deposition. Behavioral testing was performed between 4 and $5 \mathrm{~h}$ after drug application in a soundproof chamber maintained at $30^{\circ} \mathrm{C}$. For stimulation experiments, female grasshoppers were transferred to a sound permissive cage containing blades of wheat and were left there to acclimate for $5 \mathrm{~min}$ before acoustic stimulation began. The acoustic stimulus was a digitized recording of a typical C. biguttulus male calling song, consisting of three song sequences (entire duration $10 \mathrm{~s}$ ), which was presented five times with $5 \mathrm{~s}$ pauses in between. Acoustic stimuli were delivered at an intensity of $65 \mathrm{~dB} /$ SPL via the software LabView (Version 7.1, National Instruments) and a loudspeaker (SoundCraft Conrad DT 25P) positioned at a distance of $15 \mathrm{~cm}$ from the female grasshoppers. The subject's behavior was monitored with a digital camera (Canon Digital Camcorder MV 10) over the first 5 min following the first acoustic stimulus. To determine the number and duration of female song sequences, the recordings were viewed on a monitor. Analysis of the videos was conducted blind to subject group (AG-treated versus saline-treated grasshoppers).

Evaluation and statistical analysis of experimental results

Analysis of pharmacological brain stimulation experiments was performed using relative values calculated by setting the longest duration of all songs stimulated at a particular injection site as $100 \%$. Potential changes in the duration of muscarine-induced stridulation following the injection of SNP were evaluated with the non-parametric Friedman test for multiple dependent values followed by the Wilcoxon-Wilcox test to identify the significantly different data sets. Comparison of duration and latency of muscarine- and NOS inhibitor-stimulated stridulation at identical stimulation sites in the grasshopper brain was performed with the Wilcoxon matched pairs signed rank test. The same test was used for evaluation of male songstimulated song duration in female grasshoppers treated alternately with AG and saline on consecutive days. Experiments involving two different treatment groups of females (e.g., treated exclusively with AG or saline) exposed to male songs were evaluated with the Wilcoxon-MannWhitney $U$-test. To provide an estimate of the variance of the stimulated song durations and latencies, standard deviations of the means were calculated and included into the histograms. Calculations were made with Excel (version 9, Microsoft) and figures assembled with PHOTOSHOP (Adobe systems). 
Anti-citrulline immunocytochemistry

To visualize citrulline as a marker for NOS activity in the grasshopper brain, we followed a modified protocol of Martinelli and coworkers (Martinelli et al. 2002). Brains of female $C$. biguttulus were extracted and fixed for $3 \mathrm{~h}$ at room temperature with $4 \%$ paraformaldehyde and $1 \%$ glutaraldehyde diluted in phosphate buffer (PB). After fixation and washing in $\mathrm{PB}$, the brains were embedded in $5 \%$ gelatine (Biomol) and sectioned with a vibrating blade microtome (50 $\mu \mathrm{m}$; Leica VT $1000 \mathrm{~S})$ in horizontal (referring to the neural axis) or sagittal direction. The sections were incubated for $10 \mathrm{~min}$ with $10 \%$ sodium borohydrate (Sigma) to reduce tissue autofluorescence, washed several times with PB containing 1\% Triton X-100 (Sigma) and exposed to blocking buffer (PB with $1 \%$ Triton X-100, $0.25 \%$ bovine serum albumin (MP Biomedicals Inc.) and $10 \%$ normal goat serum (Amersham Biosciences) for $2 \mathrm{~h}$. Tissues were exposed to a monoclonal mouse anti-citrulline antibody (Holstein et al. 2001; Martinelli et al. 2002) diluted 1:20 in blocking buffer for 2 days at $4^{\circ} \mathrm{C}$. After washing, the brain sections were incubated for 1 day in an Alexa 488-coupled secondary antibody developed in goat (Mobitec) diluted 1:300 in blocking buffer. Finally, brain sections were transferred to a 1:1 mixture of PB and glycerol and mounted on a slide for microscopic analysis. Immunofluorescence was analyzed with a confocal microscope (Leica TCS SP2) using the Leica confocal software. Brightness and contrast of images were adjusted with Image J (NIH) or Photoshop (Adobe).

\section{Anti-universal NOS immunocytochemistry}

Dissected grasshopper brains were immersion-fixed overnight in $4 \%$ paraformaldehyde in $\mathrm{PB}$, and then sectioned and further processed as described above. An anti-rabbit uNOS antiserum (Affinity BioReagent) was used as the primary antiserum, diluted 1:100 in blocking buffer. The secondary Alexa 488-coupled antibody developed in rabbit (Mobitec) was applied at 1:300 dilution.

\section{Results}

Our previous studies on restrained male $C$. biguttulus and other grasshopper species demonstrated that sound production, stimulated by activation of muscarinic acetylcholine receptors (mAChRs) in the central body, can be suppressed by co-application of various drugs activating the NO/cGMP signalling pathway (Wenzel et al. 2005). These functional observations were supported by morphological studies revealing the presence of both NO-generating (detected by anti-universal NOS immunolabeling and NADPH-diapho- rase histochemistry) and NO-responsive (cGMP accumulating) neural processes in the central body neuropil. The data suggested that endogenous NO/cGMP signalling contributes to the cephalic control of grasshopper sound production. In the present study, we have extended this approach both to grasshopper females, in order to study the contribution of brain NO signaling to the control of reproduction-related sound production in a more natural behavioral assay with unrestrained grasshoppers. In addition, we describe a subgroup of NOS expressing pars intercerebralis neurons that, when grasshoppers are restrained, accumulate citrulline and hence release NO into the central body. These neurons may generally inhibit sound production in unfavorable situations.

Endogenous release of NO in the central body inhibits sound production of female grasshoppers

In prior studies, three different drugs have been used to inhibit NOS in female grasshoppers when dissolved at $10^{-3} \mathrm{M}$ concentration in grasshopper saline and injected into central protocerebral regions at sites where muscarine $\left(10^{-3} \mathrm{M}\right)$ repeatedly elicited sound production. S-methylL-thiocitrulline and $\mathrm{N}^{G}$-nitro-L-arginine exerted variable effects on the duration of muscarine-stimulated sound production. While in some experiments, muscarine-stimulated singing was prolonged, most applications had no effect and an evaluation of all experiments conducted with these two NOS inhibitors revealed no significant changes of muscarine stimulation effects. $\mathrm{N}^{G}$-nitro-L-arginine, but not S-methyl-L-thiocitrulline, was capable of releasing sound production alone at 3 of 15 injection sites where muscarine was an effective stimulator (data not shown). In contrast, injections of AG hemisulfate salt into female $C$. biguttulus brains reliably stimulated series of song sequences or potentiated the effects of muscarine stimulation when the substances were co-injected. Figure 1a shows a complete response to an AG stimulus consisting of individual sequences of 1.5-2 s duration with the typical pattern of sound-generating hindleg movements [Fig. $1 \mathrm{~b}$ is an enlargement of the far right sequence in Fig. 1a; songs of C. biguttulus females have been described by von Helversen and von Helversen (1997)]. To compare the potency of AG-mediated disinhibition of sound production with the stimulatory effect of muscarine, which has been used as a reference in previous studies, both drugs were injected consecutively to identical sites within the central brains of 11 female grasshoppers. We detected no differences in the general movement patterns, the sequence-to-pause composition (not shown), or the duration of sound production (Fig. 1c upper histogram) between muscarine- and AGinduced songs. In contrast, AG elicited stridulatory hind leg movements after shorter latencies than muscarine at all 


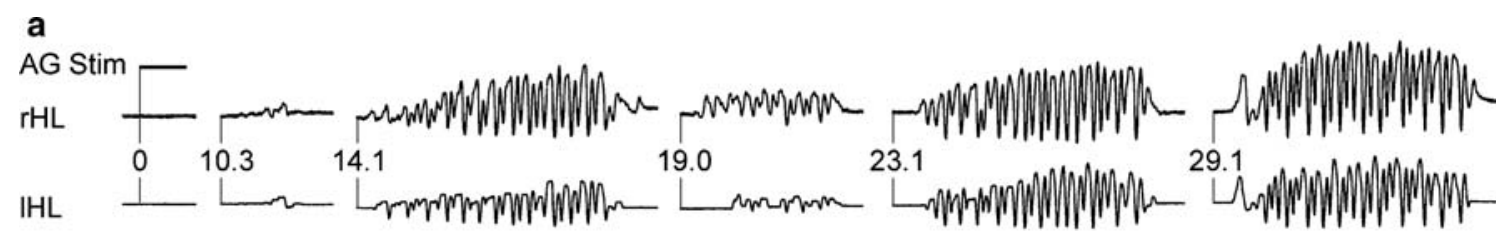

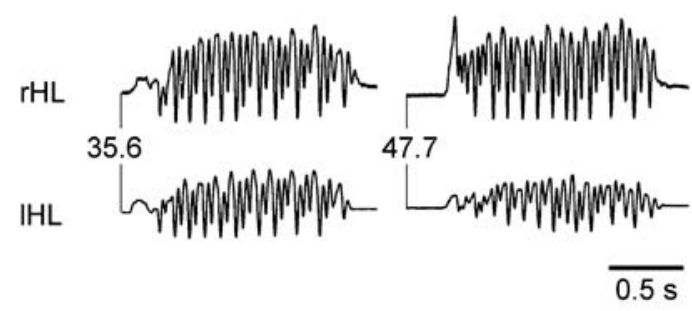

C
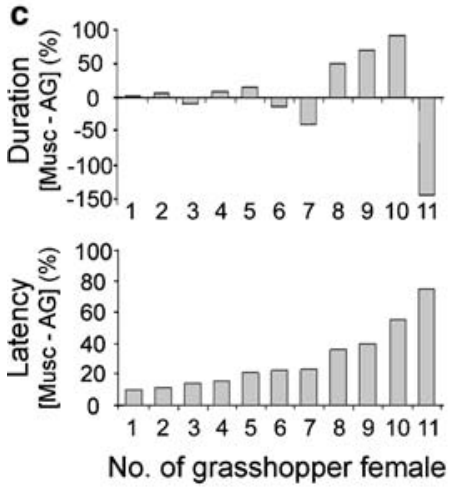

d

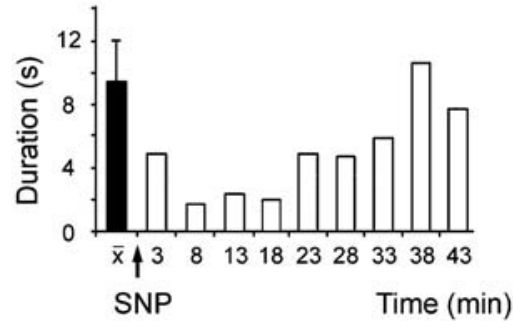

Muscarine before SNP

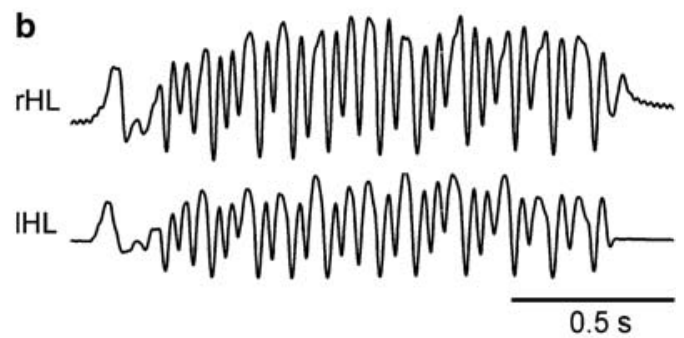

e

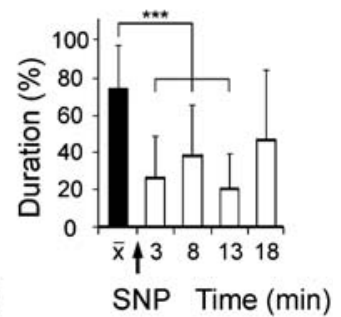

$\square$ Muscarine after SNP
Fig. 1 Pharmacological stimulation of sound production in restrained female grasshoppers. a Sound generating movements of the right (rHL) and left (lHL) hind leg stimulated by a single injection of aminoguanidine $\left(A G ; 1-3 \mathrm{nl} ; 10^{-3} \mathrm{M}\right)$ into the central body. Numbers at the beginning of each song sequence indicate time after stimulus application. b Enlarged presentation of the song sequence starting at $29.1 \mathrm{~s}$ after aminoguanidine injection shows the typical pattern of hind leg movement in Ch. biguttulus females. c Comparison of duration and latency of sound production stimulated by single injections of muscarine and aminoguanidine (both $1-3 \mathrm{nl} ; 10^{-3} \mathrm{M}$ ) into the same site within the central body of 11 female grasshoppers. While both drugs stimulated sound production of similar duration, aminoguanidine elicited the behavior at shorter latencies than did muscarine (Wilcoxon matched pairs signed rank test). d, e Reversible inhibition of muscarine-stimulated sound production by single injections of sodium nitroprusside $\left(S N P ; 10^{-3} \mathrm{M}\right)$ into the central body. d Single experiment. e Analysis of eight experiments with the non-parametric Friedman test for multiple dependent values followed by the Wilcoxon-Wilcox test to identify the significantly different data sets

muscarine-stimulated singing by exogenously applied NO, suggests that the balance of excitation and inhibition in the central body is responsible for the initiation of female grasshopper sound production.

Pharmacological inhibition of NOS promotes malestimulated sound production in female grasshoppers

In order to study the influence of endogenous NO signaling on grasshopper sound production, we injected the NOS inhibitor AG $\left(30 \mu \mathrm{l} ; 10^{-2} \mathrm{M}\right)$ into the hemolymph of receptive $C$. biguttulus females and compared their responsiveness to acoustic stimulation with conspecific male songs to saline-injected controls. Two groups of eight experimental and eight control females were injected with AG or saline at the same time on ten consecutive days and stimulated with a recording of male song between 4 and $5 \mathrm{~h}$ after 
injections. Since grasshopper song performance is known to depend on weather conditions and circadian rhythms, it was important to conduct the experiments with both treatment groups at same time. For each day, the cumulative duration of male song-stimulated sound production was determined for the groups of AG-injected and salineinjected females. As shown in Fig 2, cumulative male songstimulated sound production was higher in AG-injected females than in saline-injected controls on all experimental days. Comparison of all values obtained over the entire experimental period revealed an average singing duration of $21.7 \pm 7.9 \mathrm{~s}$ in AG-treated and $12.9 \pm 6.8 \mathrm{~s}$ in salineinjected females. Both treatment groups responded best on experimental days 5-7 probably due to outside weather conditions. Statistical analysis with a rank based $U$-test (ranks are indicated in Fig. 2) revealed a highly significant difference of male song-stimulated sound production between AG-treated and saline-injected groups of females ( $P \leq 0.01 ; U$-test after Wilcoxon-Mann-Whitney).

Although female grasshoppers used in the experiment emerged from the same clutch and were raised under exactly the same conditions, individual differences in sound production were noted between animals within both AGand saline-treated groups. To exclude bias resulting from assigning endogenously more active singers to one of the treatment groups, we conducted another experimental series in which each individual was alternately injected
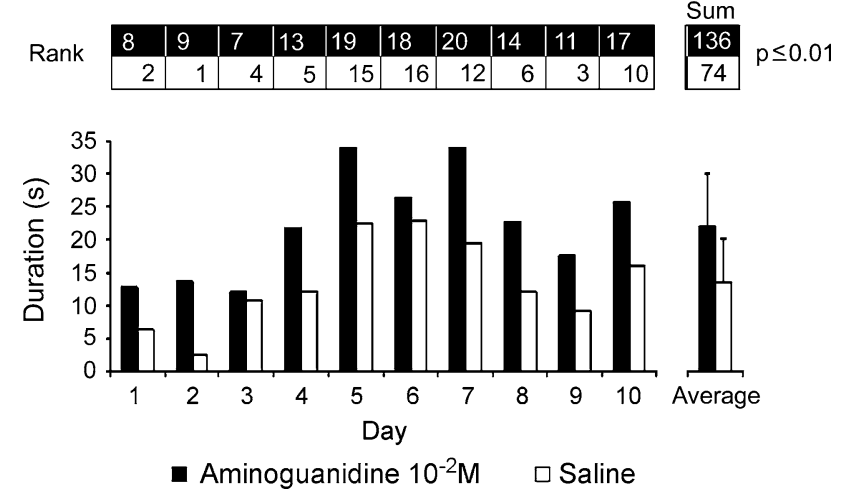

Fig. 2 Male song-stimulated sound production in aminoguanidine $(A G)$ - and saline-injected female grasshoppers. Two groups of eight C. biguttulus females were stimulated with calling songs of conspecific males at 4-5 h after receiving an injection of $30 \mu \mathrm{l} \mathrm{AG}\left(10^{-2} \mathrm{M}\right)$ or saline on ten consecutive days. Each column in the main graph represents the sum of the responses of all eight females on the respective experimental day. Male song-stimulated sound production was higher in AG treated females (black columns) than in saline-injected controls (white columns) on each experimental day. This difference is also reflected in the average daily response over the entire experimental period (right). Comparison of all female responses over the entire experimental period with a non parametric rank-based test (ranks indicated above main graph) revealed a highly significant difference $(P \leq 0.01 ; U$-test after Wilcoxon-Mann-Whitney) in the average duration of sound production between AG- and saline-treated grasshopper females with AG and saline on four consecutive days. Ten females received injections of $\mathrm{AG}$ on the first experimental day while nine females of the same age started with saline injections, enabling behavioral testing of similar numbers of animals from both groups under the same weather conditions. The longest duration of male song-stimulated sound production of each individual female recorded on one of the four experimental days was set as $100 \%$ and the relative duration of sound production on the other 3 days was calculated accordingly. Of the 19 females tested, 15 produced more prolonged response songs after AG injection compared to saline injections while four were less responsive following AG treatment (Fig. 3). On average (all 19 females included), the females stridulated $34.9 \%$ longer after AG treatment (63.4\%) than after saline (28.5\%), which was detected as a statistically significant difference $(P \leq 0.01)$ by the Wilcoxon matched pairs signed rank test. Comparison of these experimental values as two treatment groups (AG-injected versus saline-injected) by $U$-testing also revealed a significant difference in the duration of male song-stimulated female sound production $(P \leq 0.025$, data not shown). Within each group of females (group 1 started experimental series with $A G$ injection on the first day while group 2 began with saline-injection), the duration of sound production on days with AG treatment was always higher than on days with saline treatment and no difference in the total duration of sound production on all four experimental days was detected between the two groups (data not shown).

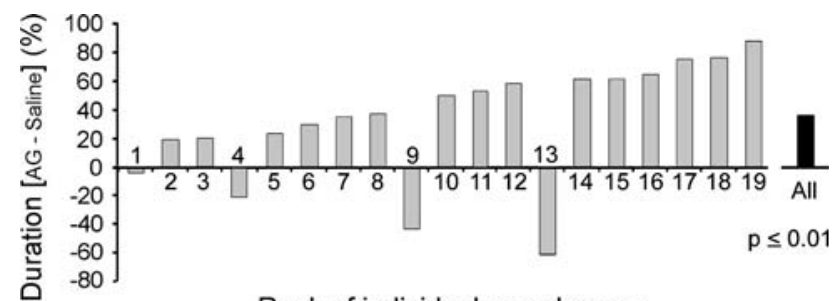

Rank of individual grasshopper

Fig. 3 Male song-stimulated sound production in aminoguanidine $(A G)$ - and saline-injected female grasshoppers. Two groups of C. biguttulus females were injected alternately with $30 \mu \mathrm{AG}\left(10^{-2} \mathrm{M}\right)$ and saline, and stimulated with male calling songs, on four consecutive days. One group started with AG treatment on day 1 while the other started with saline treatment. There was no difference in the overall responsiveness to male song between the two groups with different starting treatment. The graph displays the differences of male songstimulated sound production following AG (sum of 2 days) and saline injection (sum of 2 days) for 19 individual female grasshoppers. Sixteen of the females were more responsive to male song after AG treatment than after saline injections [highly significant difference $(P \leq 0.05)$ in Wilcoxon's matched pairs signed rank test]. Comparison of the responses from all experiments with the 19 females over the entire experimental period revealed a significant difference between the duration of sound production following AG treatment and its duration after saline injections $(P \leq 0.025$; $U$-test after Wilcoxon-MannWhitney; data not shown) 
Systemic application of AG enhanced male song-stimulated sound production of receptive females in groups of females repeatedly treated with AG or saline and in individual females treated alternately with AG and saline. These results suggest that AG-mediated inhibition of endogenous NOS function may reduce the inhibitory effect of endogenous NO signalling on sound production.

NOS-containing central complex neurons accumulate citrulline in behavioral situations that suppress sound production

Inhibition of NOS in the central body is sufficient to release sound production in restrained grasshoppers of both genders (shown for C. biguttulus females in Fig. 1a, b). Being restrained in the experimental setup is undoubtedly a situation in which sound production would be an inappropriate behavior and hence should be suppressed. We hypothesized that the release of NO in the central body might mediate suppression of sound production in unfavorable situations and used anti-citrulline immunocytochemistry to label preceding activation of NO-producing neurons in the brain. Citrulline is generated as a byproduct during NO production and its accumulation can be used as a marker for NOS activation during the period preceding fixation of the central nervous tissue.

Strong accumulation of citrulline was detected in NOScontaining cell bodies of pars intercerebralis neurons and their projections within the upper division of the central body (Fig. 4). Figure 4a illustrates the positions of NOScontaining neuronal cell bodies in the pars intercerebralis. As described previously (Kurylas et al. 2005; Wenzel et al. 2005), the anti-universal NOS serum used for visualization of NOS produced only diffuse staining of cell bodies and neurites against a rather intense unspecific background. In one brain, up to 30 of the NOS-containing cell bodies located in both the lateral and medial portions of pars intercerebralis were intensely labeled by citrulline immunocytochemistry. The neurites of these columnar neurons either remained ipsilaterally or projected as large diameter neurites via four pairs of fiber bundles ( $\mathrm{w}, \mathrm{x}, \mathrm{y}, \mathrm{z}$ bundles) through the posterior chiasm to the contralateral side before entering columns of the central body upper division. Here they divided into fine arborizations that appeared to overlap with the projection areas of neurons from adjacent columns (Fig. 4b, c). Based on their "hook"-like appearance (best seen in Fig. 5e), some of the projections are likely to originate from pontine neurons, intrinsic neurons of the central body that have their somata in more medial regions of the pars intercerebralis. Sagittal sections through the central body revealed that the fine arborizations were restricted to layers II and III of the upper division (Fig. 4d) and no citrulline staining was detected in the noduli and the lower division (Fig. 4c, d). In addition to columnar neurons, up to three pairs of cell bodies in the ventro-median protocerebrum were consistently labeled (arrowheads in Fig. 4c). These most likely represent a subgroup of tangential neurons of the central body, which have previously been described morphologically (Homberg et al. 1999; Kurylas et al. 2005), although citrulline-immunoreactive projections into the lower division were not detected. As seen in the sagittal section (arrowheads in Fig. 4d), their neurites enter the central body through the posterior groove. Their projections intermingle with arborizations of citrulline-immunopositive pars intercerebralis neurons in layers II and III of the central body upper division and thus could not be described in detail in the present study. These results indicate that two types of central complex neurons, a group of pars intercerebralis neurons and up to three pairs of tangential neurons, actively release NO into the upper division of the grasshopper central body under conditions of restraint.

Pharmacological inhibition of NOS prevents accumulation of citrulline in central complex neurons

Both the perineural sheath and glia have been demonstrated to limit the transfer of endogenous chemical signals and applied drugs between the hemocoel and central nervous neuropils. In order to test whether the increased responsiveness of female grasshoppers to male song stimulation following injection of AG into the hemolymph is mediated by reduced NOS activity in the central nervous system (especially in the central body), we performed anti-citrulline immunocytochemistry on brain sections of AG- and salineinjected $C$. biguttulus females. Animals were treated in the same way as during the behavioral experiments described above and their brains were dissected in fixative $4 \mathrm{~h}$ after AG or saline application. All specimens to be directly compared with each other were processed with the same solutions for identical time periods and scanned with identical settings at the confocal microscope.

A large overall reduction in citrulline immunoreactivity was detected in all intact brain neuroils of AG-treated females compared with saline-injected controls. Since NO signalling is known to contribute to the processing of olfactory and visual sensory information, we evaluated the antennal lobes and the optic lobes in addition to the central complex for differences in citrulline-associated immunofluorescence. The antennal lobes of saline-injected females contained intensely stained cell bodies of olfactory interneurons and a fine meshwork of intensely stained neurites throughout the entire glomerular region (Fig. 5a). In contrast, antennal lobes of AG-treated females contained fewer and weakly labeled cell bodies and citrulline accumulation in the glomerular neuropil was barely visible only in some regions (Fig. 5b). No such differences in staining intensity were 

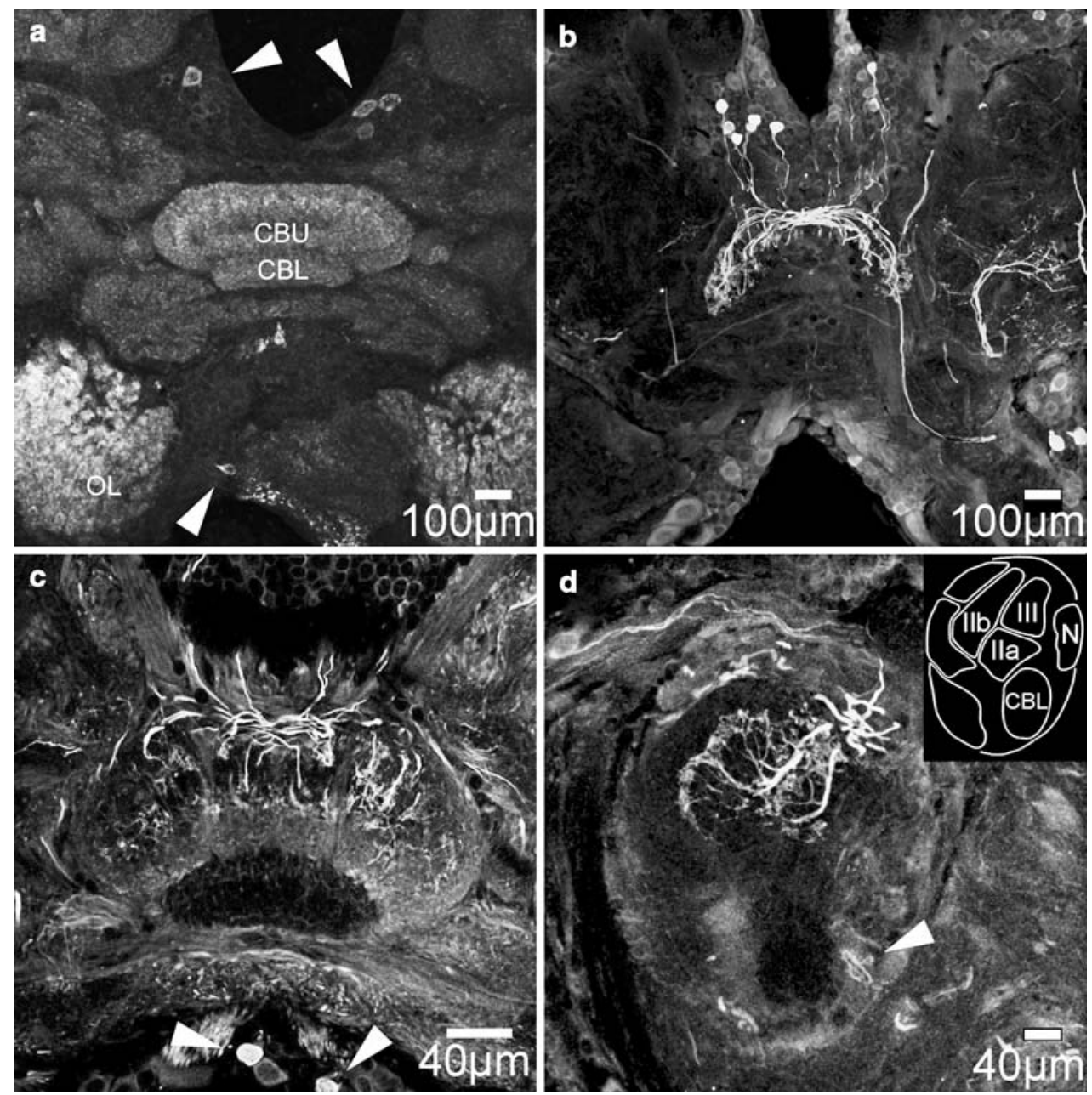

Fig. 4 Expression of nitric oxide synthase (NOS) and accumulation of citrulline in the brain of female $C$. biguttulus. a Horizontal brain section processed for anti-universal NOS immunocytochemistry containing labeled cell bodies in the pars intercerebralis region and the ventromedian protocerebrum (arrowheads) and diffuse staining in the upper division of the central body (CBU) and the glomeruli of antennal lobes $(\mathrm{OL})$. b, $\mathbf{c}$ Horizontal brain sections processed for anti-citrulline immunocytochemistry resulted in strong labeling of cell bodies in the pars intercerebralis region and their projections into the upper division of the central body and the lateral accessory lobes. Immunopositive primary neurites either remained ipsilaterally or crossed through the

detected in the optic lobes, were NO is thought to act as a retrograde transmitter at the photoreceptor-to-monopolar cell synapse in the lamina (Elphick et al. 1996; Schmachtenberg and Bicker 1999). In both AG- and saline-injected females, intense citrulline-associated immunofluorescence was apparent in cell bodies and fibers of laminae (Fig. 5c, d). However, strong and AG-insensitive activation of NOS in the lamina may be a consequence of mechanical damage to this neuropil during dissection of the brain, rather than represent a physiologically relevant activation. Similarly, injury to peripheral visual neuropils in locusts has been demonstrated to amplify NO-stimulated accumulation

posterior chiasm to the contralateral side before entering the central body. These neurons represent columnar neurons of the central body. Arrowheads in $\mathbf{c}$ indicate intensely labeled cell bodies in the ventromedian protocerebrum whose neurites enter the central body through the posterior groove (arrowhead in d) between the lower division $(\mathrm{CBL})$ and the noduli $(N)$. d Parasagittal section through the central body and schematic map of transected neuropils. Citrulline immunolabeled neurites innervate layers II and III of the central body upper division. The lower division of the central body is entirely devoid of citrulline-associated immunolabeling (also visible in c)

of cGMP in photoreceptors and visual interneurons, an observation that has been attributed to enhanced NO release from compromised tissues (Seidel and Bicker 2002).

Aminoguanidine treatment induced a marked reduction in the accumulation of citrulline in the central complex (Fig. 5f, h). Staining was entirely absent from cell bodies of pars intercerebralis columnar neurons and tangential neurons in the ventro-median protocerebrum. Citrulline immunopositive neurites projecting through columns of the central body upper division and their arborizations in layers II and III of the neuropil were detected in smaller numbers and with 
Fig. 5 Anti-citrulline immunocytochemistry on horizontal sections through brain regions of saline- and aminoguanidine (AG)-injected C. biguttulus females. a Accumulation of citrulline in olfactory interneurons of the antennal lobe. Both cell bodies and their glomerular projections show intense immunolabeling in saline-injected females. b Anti-citrulline staining is weaker in cell bodies of olfactory interneurons and almost absent from most parts of the glomerular neuropil in AG-treated females. c, d No difference in the intensity of citrulline-associated immunofluorescence was detected in cell bodies and neurites of monopolar cells in the lamina neuropil of the optic lobes. e-h Brain sections through the central body. e, $\mathbf{g}$ Two sections from the same preparation with strong citrulline-associated immunofluorescence in columnar neurons of the central body of saline-injected females. Cell bodies in the pars intercerebralis and their projections into the upper division of the central body are clearly labeled. $\mathbf{f}, \mathbf{h}$ Cell bodies of columnar neurons and their projections into the central body were essentially unstained in aminoguanidine-treated animals. As shown in two sections from the same preparation, these animals had fewer and more weakly labeled projections than saline-injected controls

\section{Saline}
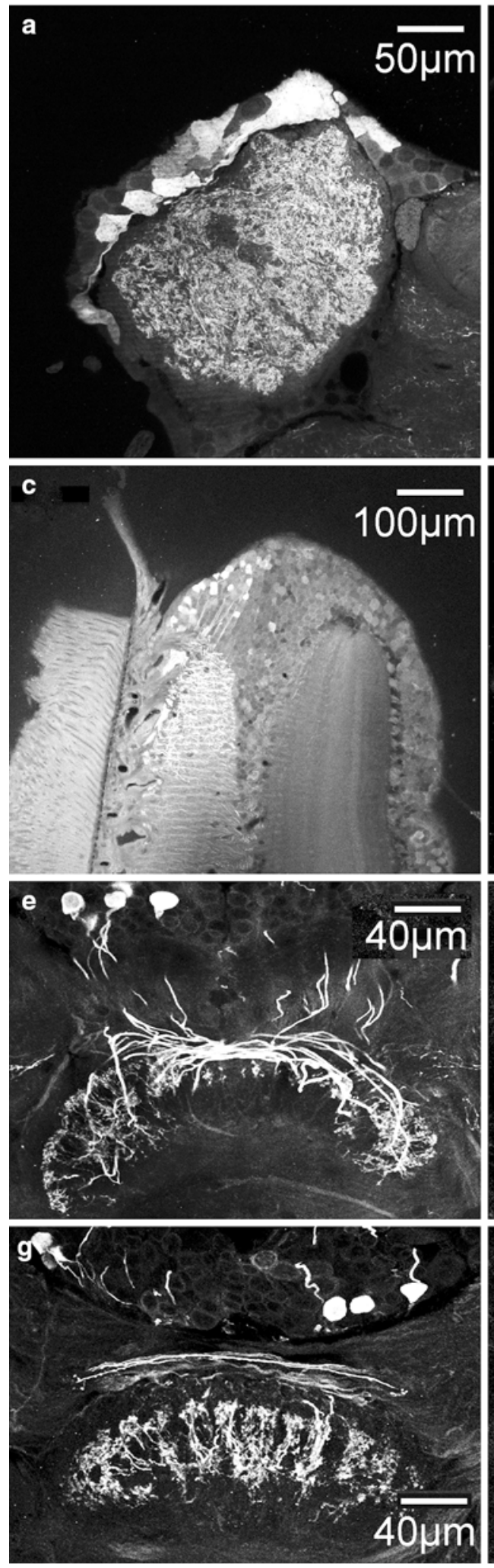

Aminoguanidine
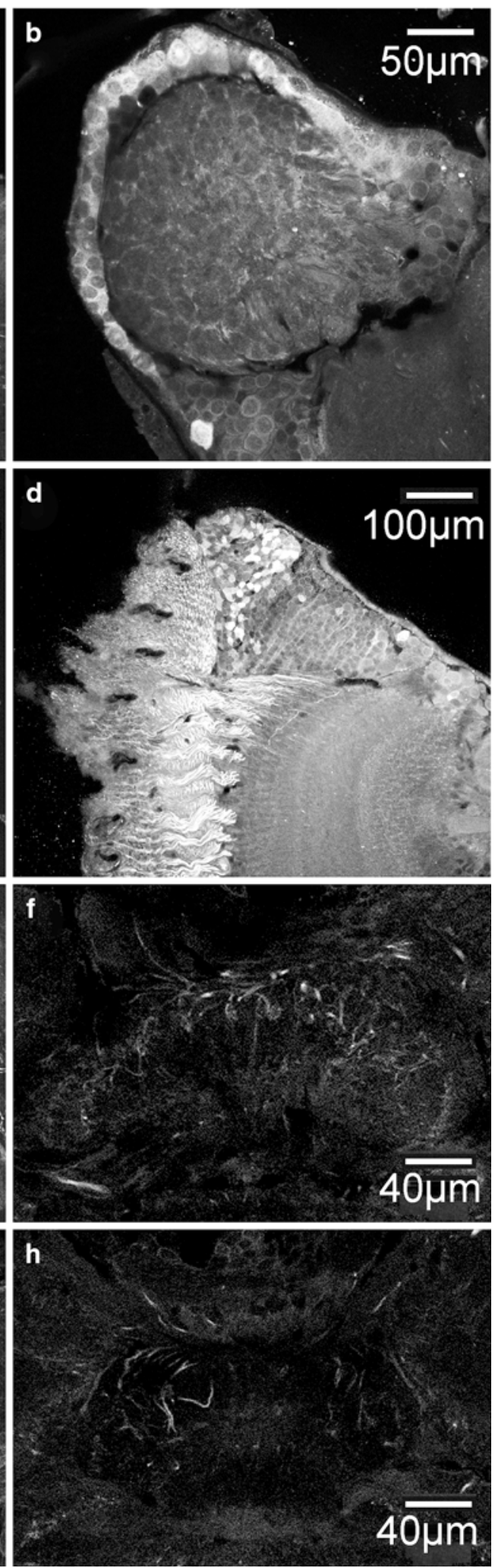

less intense labeling in all AG-treated preparations directly compared to identically processed tissues from saline-injected controls (Fig. 5e, g compared with Fig. 5f, h). In brains of
AG-treated female grasshoppers, weak citrulline accumulation was only detected in the large diameter neurites projecting through the periphery of the central body upper division, 
while most small diameter arborizations in layers II and III were devoid of staining (as shown in Fig. 5f, h). These results indicate that injection of AG into the hemolymph effectively inhibits NOS activity in the grasshopper brain and reduces basal NO release from pars intercerebralis columnar and tangential neurons in the central body.

\section{Discussion}

The present study represents the first pharmacological effort to understand the signalling mechanisms contributing to the cephalic control of sound production in female grasshoppers. We provide evidence that NO/cGMP signalling in the central complex suppresses sound production and we identify candidate neurons that mediate this suppression based upon their NO-releasing activity during exposure to unfavorable behavioral situations in which sound production would be inappropriate.

The central complex of insects is largely concerned with (pre-) motor processing and is widely regarded as a "higher decision center" for the selection, initiation, maintenance and modulation of behavior (Strauss 2002; Popov et al. 2005; Wessnitzer and Webb 2006; Ridgel et al. 2007). In line with studies on D. melanogaster mutants with structural impairments of the central complex (Popov et al. 2005), previous studies on acoustically communicating grasshoppers indicated that the central complex functions as the major center for the initiation and coordination of situation-specific sound patterns (Heinrich et al. 2001). Neural processing within the central complex neuropil precedes the activation of stridulatory command neurons that connect decision making centers in the protocerebrum with the central pattern generating circuits in thoracic ganglia, which in turn drive the hind legs to produce specific sound patterns (Hedwig 1994; Hedwig and Heinrich 1997; Heinrich 2002). Pharmacological studies on restrained but otherwise intact male grasshoppers identified a number of signalling pathways in the central complex (transmitters, modulators, receptors, intracellular second messenger pathways) that promote or suppress sound production (Heinrich et al. 1997, 1998; Wenzel et al. 2005). NO was demonstrated to suppress muscarine-stimulated singing via activation of sGC and production of cGMP. Together with the detection of NOS in the central body upper division and NO-stimulated accumulation of cGMP in the lower division, the NO/cGMP signalling pathway was suggested to play a major role in the control of grasshopper sound production.

\section{Studies on restrained female grasshoppers}

Although sound production in female grasshoppers underlies control mechanisms related to reproductive status
(Loher and Huber 1964; von Helversen and von Helversen 1997), it can be reliably stimulated by injections of muscarine into the central body in both recently mated and non-mated receptive females (Heinrich et al. 1997). In the present study, we first confirmed that experimental release of NO in the central body inhibits muscarine-stimulated sound production in restrained C. biguttulus females with similar reliability, reversibility and time course as in males of the same species (Fig. 1; Wenzel et al. 2005). Moreover, inhibition of NOS by injection of AG into the central body appeared to be sufficient to release sound production, proving that singing is suppressed in this situation by basal NO production in this neuropil. Movement patterns and temporal structure of sequences and pauses of AG-induced songs were similar to muscarine-stimulated and natural songs of this species. This contrasts with results obtained by disinhibition of sound production mediated by chloride channelassociated signalling pathways (e.g., GABA and glycine) using picrotoxin, which induced mixtures of song patterns with irregular temporal compositions in males of various species that neither occur after muscarine stimulation nor in natural behavior (Heinrich et al. 1998). The similarity between muscarine-stimulated and AG-induced sound production suggests that both muscarinic cholinergic excitation and NO/cGMP-mediated inhibition may affect the same processing pathway within the central complex and influence its output activity according to the situation. Restraint, a situation in which sound production is unfavorable, may therefore cause suppression of singing through activation of NOS-containing neurons in the central body. In fact, restraint-dependent inhibition is sufficiently strong to suppress male calling songs during stimulation with the song of a conspecific female. Female songs are known to represent very strong sensory stimuli that release immediate reactions and deviations from ongoing behavior in unrestrained males. Although female songs have been demonstrated to stimulate cholinergic pathways in the central body (Hoffmann et al. 2007), this excitation is obviously not sufficient to surmount restraint-mediated inhibition. However, this inhibition can be overcome by injections of muscarine into the central body, sufficient to stimulate sound production in the restrained grasshoppers.

In order to identify the neurons responsible for suppression of sound production in inhospitable situations, citrulline immunocytochemistry was applied to grasshopper brains. Citrulline has been shown to accumulate in the cytosol of NO-producing cells, where it is generated in equal numbers to NO molecules. Accumulation of citrulline has been used as a marker for recent NOS activation prior to fixation of tissues (Holstein et al. 2001; Martinelli et al. 2002; Cayre et al. 2005), although its half life after its formation in the cytosol is unknown and estimations about the period of its production prior to tissue fixation are difficult 
to make. Although a large number of neurons have been labeled by NOS immunocytochemistry and NADPH diaphorase staining in all neuropils of the locust and cockroach central complex (Ott and Elphick 2002; Kurylas et al. 2005), citrulline immunoreactivity in the central complex of restrained grasshopper females appeared to be restricted to particular regions of the central body upper division and was sufficiently distinct to enable the identification of the responsible neurons (Figs. 4,5). These central complex neurons, up to 30 cell bodies in the pars intercerebralis and up to six cell bodies in the ventro-median protocerebrum, accumulate citrulline and release NO prior to tissue fixation, when grasshoppers are restrained for extraction of their brains. Based on the positions of their cell bodies, their large diameter neurites in the columns and their arborizations in layers II and III of the central body upper division, the pars intercerebralis neurons resembled columnar and pontine neurons whose anatomy has previously been described in Schistocerca gregaria (Kurylas et al. 2005). Columnar neurons of this locust species are activated by particular oscillation planes of polarized light, forming a map-like system for zenithal E-vector representation (Heinze and Homberg 2007). In C. biguttulus columnar neurons of similar anatomical type have been shown to be the only neurons that express detectable levels of muscarinic ACh receptors (Hoffmann et al. 2007). Since injection of muscarine into the central body can stimulate sound production and increase responsiveness to conspecific songs, these columnar neurons seem to play a decisive role for the initiation of grasshopper acoustic communication. Whether the same types of neurons process information related to both polarization-based spatial orientation in $S$. gregaria and acoustic communication in C. biguttulus, or subtypes of columnar neurons are dedicated to different sensorimotor tasks, will be important information to gain for understanding the general function of the central complex and the mechanisms by which it selects particular behaviors for execution. Since NO released from central complex neurons suppresses muscarine-stimulated sound production in grasshoppers and columnar neurons that express the muscarinic ACh-receptor are output neurons of the central complex (Vitzthum et al. 2002; Homberg 2004), these columnar neurons may represent direct or indirect targets of NOmediated inhibition. In order to identify the sensory pathways and presynaptic neurons that activate NO-releasing central complex neurons, we will attempt to identify their postsynaptic receptors and the expression of the corresponding transmitter in the central complex by immunocytochemistry and evaluate whether deprivation of particular sensory input modulates situation-specific suppression of sound production. The projections of citrulline-immunoreactive cells in the ventro-median protocerebrum could not be traced in detail, and no match to previously described neurons could be discerned. Anatomical studies in the locust brain revealed various types of NADPH diaphorasepositive tangential neurons whose cell bodies are located in the ventro-median protocerebrum and whose neurites enter the central complex via the posterior groove (Müller et al. 1997). Two types of these tangential neurons arborise in the lower division of the locust central body (Kurylas et al. 2005), which was free of citrulline-immunoreactivity in our studies, suggesting that such neurons either do not exist in C. biguttulus or are not active in the situation preceding brain dissection and tissue fixation. A third type of tangential neuron innervating the upper division of the central body was demonstrated to contain the transmitter GABA (Homberg et al. 1999). However, we did not observe colocalization of GABA and citrulline in any of the somata of the ventro-median protocerebrum (data not shown).

\section{Studies with unrestrained female grasshoppers}

In order to test whether NO signaling contributes to the control of female reproductive behavior by modulating responsiveness to the conspecific male song, we injected female grasshoppers in the state of active copulatory readiness with the NOS inhibitor AG. Females respond to male calling songs or even sing spontaneously only after prolonged periods without copulation (Loher and Huber 1964). In the species $C$. biguttulus used in this study, males and females are known to perform alternating song dialogues (von Helversen and von Helversen 1997). Also, in addition to the reproductive state of females, sound production in both sexes depends on weather conditions, intensity of illumination, temperature and the circadian rhythm. It is therefore important to conduct behavioral studies with different experimental groups of grasshoppers at the same time. Female sound production in response to male callings songs was monitored on ten consecutive days and compared between AG-injected animals and saline-injected controls. On each day, AG-treated females showed increased responsiveness to the behaviorally meaningful stimulus in comparison with controls (Fig. 2). Although all tested females were derived from the same clutch, we noticed individual differences in singing activity over the entire experimental period. To exclude undesirable bias from assigning animals to the treatment or control group, we conducted a second series of experiments in which each female was alternately injected with $\mathrm{AG}$ or saline on four consecutive days. We found that 15 out of the 19 tested females produced more response songs to male song stimulation (Fig. 3) and confirmed that the results of the first series were due to pharmacological treatment rather than to individual differences in singing activity between females.

In addition to its signalling function in insect central nervous systems, NO has been demonstrated to affect heart 
rate (Bullerjahn et al. 2006), neuromuscular excitation (Wildemann and Bicker 1999; Zayas et al. 2000; Zayas and Trimmer 2007) and neurosecretory release (Zayas et al. 2000; Bullerjahn and Pflüger 2003; Wicher et al. 2004). These tissues and functions may have been modulated by the systemic application of AG to the grasshopper hemolymph, and a direct or indirect impact on general activity and the performance of sound production, either promotional or inhibitory, cannot be excluded. Since the ganglionic sheath and peripheral layers of glia have been shown to prevent effective translocation of various systemically applied drugs and bath applied staining reagents into the central nervous system (Stumpner 1998), we were uncertain whether AG, after its injection to the abdominal hemolymph, would reduce NO production in the brain. However, efficient inhibition of NOS activity in the brain was demonstrated by the striking reduction of citrulline accumulation in all brain regions that remained intact between brain extraction and tissue fixation (Fig. 5). As the only exception, citrulline accumulation was not reduced by AG administration in cell bodies and neurites of laminae, which was most likely caused by injury to this neuropil during brain extraction. A similar injury-related activation of NO/cGMP signaling pathways has been observed in the locust visual system (Seidel and Bicker 2002). Since accumulation of citrulline in the distal optic lobes does not result from particular sensory stimulation, matching intensities of citrulline-associated immunofluorescence can be regarded as markers for identical processing of brain tissues from the AG- and saline-treated grasshoppers. Of greatest significance for the control of sound production, citrulline immunoreactivity was markedly reduced in central complex neurons. Especially in the central body, where experimental release of NO has been shown to suppress sound production, citrulline immunofluorescence in fine arborizations in layers II and III of control animals was essentially absent in AG-injected females. Since both dissection/fixation of brain tissues for anti-citrulline immunocytochemistry and behavioral testing occurred at similar times following injection of AG, reduced generation of $\mathrm{NO}$ in the central body and other regions can also be assumed during behavioral tests with male song stimulation. Although we do not expect such profound NO production in unrestrained female grasshoppers as appears in the central complex of restrained individuals, differences in basal NO-mediated inhibition of sound production seem to be sufficiently significant to increase the responsiveness in AG-injected individuals.

A link between sensory stimulation and increased NO signalling in higher brain centers was established in crickets reared in isolation or together with conspecifics. Crickets reared under social conditions exhibited enhanced NO production in mushroom bodies, which stimulated neurogenesis in adult cricket brains (Cayre et al. 2005). It was suggested that the functional status of NOS-, sGC- and cGMP-initiated mechanisms may reflect different physiological states in insects that impact behavioral choices. Accordingly, the situation-specific amount of basal NOS activity in the central body of female grasshoppers may determine whether excitation generated by the perception of conspecific calling songs triggers a specific response. NO production in the central body should be reduced in environmental situations, where sound production is an adequate behavior. Our attempts to conserve the motivated state have failed thus far, since any disturbance of the grasshopper should activate signalling pathways that suppress sound production, and since rapid freezing of grasshoppers in liquid nitrogen after prolonged and undisturbed stimulation with conspecific songs interferes with anti-citrulline immunocytochemistry. Whether NO production in the central complex may reflect both the actual environmental situation and the reproductive state of female grasshoppers to modulate the activation threshold for mating-related behaviors will be the subject of future studies.

Acknowledgments The studies were supported by the Deutsche Forschungsgemeinschaft (HE 3301/2), a Georg-Christoph-Lichtenberg Stipend provided by the Göttingen Masters/PhD program in Neurosciences (to Michael Kunst and Anja Weinrich) and a PostDoc stipend of the Hertie Foundation (to Michael Kunst). We thank Margret Winkler, Patricia Sprysch and Matthias Schink for valuable technical assistance and animal care and Daniela Gocht for her advice concerning statistical evaluations. The experiments comply with the "Principles of animal care", publication No.86-23, revised 1985 of the National Institute of Health and with German laws for animal care (Deutsches Tierschutzgesetz).

Open Access This article is distributed under the terms of the Creative Commons Attribution Noncommercial License which permits any noncommercial use, distribution, and reproduction in any medium, provided the original author(s) and source are credited.

\section{References}

Aonuma H, Iwasaki M, Niwa K (2004) Role of NO signaling in switching mechanisms in the nervous system of insect. SICE Annu Conf 3:2477-2482

Bausenwein B, Müller NR, Heisenberg M (1994) Behavior-dependent activity labeling in the central complex of Drosophila during controlled visual stimulation. J Comp Neurol 340:255-268

Bicker G (1998) NO news from insect brains. Trends Neurosci 21:349-355

Bicker G (2001) Sources and targets of nitric oxide signalling in insect nervous systems. Cell Tissue Res 303:137-146

Bullerjahn A, Pflüger H-J (2003) The distribution of putative nitric oxide releasing neurones in the locust abdominal nervous system: a comparison of NADPHd histochemistry and NOS-immunocytochemistry. Zoology 106:3-17

Bullerjahn A, Mentel T, Pflüger H-J, Stevenson PA (2006) Nitric oxide: a co-modulator of efferent peptidergic neurosecretory cells including a unique octopaminergic neurone innervating locust heart. Cell Tissue Res 325:345-360

Cayre M, Malaterre J, Scotto-Lomassese S, Holstein GS, Martinelli GP, Forni C, Nicolas S, Aouane A, Strambi C, Strambi A (2005) 
A role for nitric oxide in sensory-induced neurogenesis in an adult insect brain. Eur J Neurosci 21:2893-2902

Clements AT, May TE (1974) Studies on locust neuromuscular physiology in regulation to glutamic acid. J Exp Biol 60:335-378

Colasanti M, Venturini G (1998) Nitric oxide in invertebrates. Mol Neurobiol 17:157-174

Davies SA (2000) Nitric oxide signalling in insects. Insect Biochem Mol Biol 30:1123-1138

Elphick MR, Williams L, O'Shea M (1996) New features of the locust optic lobe: evidence of a role for nitric oxide in insect vision. J Exp Biol 199:2395-2407

Elsner N (1974) Neuroethology of sound production in gomphocerine grasshoppers (Orthoptera: Acrididae). I. Song patterns and stridulatory movements. J Comp Physiol A 88:67-102

Elsner N, Popov AV (1978) Neuroethology of acoustic communication. Adv Insect Physiol 13:229-335

Garthwaite J, Boulton CL (1995) Nitric oxide signalling in the central nervous system. Annu Rev Physiol 57:683-706

Hedwig B (1994) A cephalo-thoracic command system controls stridulation in the acridid grasshopper Omocestus viridulus L. J Neurophysiol 72:2015-2025

Hedwig B, Heinrich R (1997) Identified descending brain neurons control different stridulatory motor patterns in an acridid grasshopper. J Comp Physiol A 180:285-294

Hedwig B, Knepper M (1992) Neurolab, a comprehensive program for the analysis of neurophysiological and behavioural data. J Neurosci Methods 45:135-148

Heinrich R (2002) Impact of descending brain neurons on the control of stridulation, walking and flight in orthoptera. Microsc Res Tech 56:292-301

Heinrich R, Ganter GK (2007) Nitric oxide/cyclic GMP signalling and insect behaviour. Adv Exp Biol 1:107-127

Heinrich R, Hedwig B, Elsner N (1997) Cholinergic activation of stridulatory behaviour in the grasshopper Omocestus viridulus, (L.). J Exp Biol 200:1327-1337

Heinrich R, Rozwod K, Elsner N (1998) Neuropharmacological evidence for inhibitory cephalic control mechanisms of stridulatory behaviour in grasshoppers. J Comp Physiol A 183:389-399

Heinrich R, Wenzel B, Elsner N (2001) A role for muscarinic excitation: control of specific singing behavior by activation of the adenylate cyclase pathway in the brain of grasshoppers. Proc Natl Acad Sci USA 98:9919-9923

Heinze S, Homberg U (2007) Maplike representation of celestial E-vector orientations in the brain of an insect. Science 315:995-997

Hoffmann K, Wirmer A, Kunst M, Gocht D, Heinrich R (2007) Muscarinic excitation in grasshopper song control circuits is limited by acetylcholinesterase activity. Zool Sci 24:1028-1035

Holstein GR, Friedrich VL Jr, Martinelli GP (2001) Monoclonal L-citrulline immunostaining reveals nitric oxide-producing vestibular neurons. Ann N Y Acad Sci 942:65-78

Homberg U (2004) In search of the sky compass in the insect brain. Naturwissenschaften 91:199-208

Homberg U, Vitzthum H, Müller M, Binkle U (1999) Immunocytochemistry of GABA in the central complex of the locust Schistocerca gregaria: identification of immunoreactive neurons and colocalization with neuropeptides. J Comp Neurol 409:495-507

Ilius M, Wolf R, Heisenberg M (1994) The central complex of Drosophila melanogaster is involved in flight control: studies on mutants and mosaics of the gene ellipsoid body open. J Neurogenet 9:189-206

Kurylas AE, Ott SR, Schachtner J, Elphick MR, Williams L, Homberg $\mathrm{U}$ (2005) Localization of nitric oxide synthase in the central complex and surrounding midbrain neuropils of the locust Schistocerca gregaria. J Comp Neurol 484:206-223

Loher W, Huber F (1964) Experimentelle Untersuchungen am Sexualverhalten des Weibchens der Heuschrecke Gomphocerus rufus L. (Acridinae). J Insect Physiol 10:13-36
Martinelli GPT, Friedrich VL, Holstein GR (2002) L-Citrulline immunostaining identifies nitric oxide production sites within neurons. Neuroscience 114:111-122

Moroz LL (2001) Gaseous transmission across time and species. Am Zool 41:304-320

Müller U (1997) The nitric oxide system in insects. Prog Neurobiol $51: 363-381$

Müller M, Homberg U, Kühn A (1997) Neuroarchitecture of the lower division of the central body in the brain of the locust (Schistocerca gregaria). Cell Tissue Res 288:159-176

Osborne KA, Robichon A, Burgess E, Butland S, Shaw RA, Coulthard A, Pereira HS, Greenspan RJ, Sokolowski MB (1997) Natural behavior polymorphism due to a cGMP-dependent protein kinase of Drosophila. Science 277:834-836

Ott SR, Elphick MR (2002) Nitric oxide synthase histochemistry in insect nervous systems: methanol/formalin fixation reveals the neuroarchitecture of formaldehyde-sensitive NADPH diaphorase in the cockroach Periplaneta americana. J Comp Neurol 448:165-185

Ott SR, Elphick MR (2003) New techniques for whole-mount NAD$\mathrm{PH}$-diaphorase histochemistry demonstrated in insect ganglia. J Histochem Cytochem 51:523-532

Popov AV, Peresleni AI, Ozerskii PV, Shchekanov EE, SavvateevaPopova EV (2005) The role of the flabellar and ellipsoid bodies of the central complex of the brain of Drosophila melanogaster in the control of courtship behavior and communicative sound production in males. Neurosci Behav Physiol 35:741-750

Prast H, Philippu A (2001) Nitric oxide as modulator of neuronal function. Prog Neurobiol 64:51-68

Ridgel AL, Blythe EA, Ritzmann RE (2007) Descending control of turning behaviour in the cockroach, Blaberus discoidalis. J Comp Physiol A 193:385-402

Schmachtenberg O, Bicker G (1999) Nitric oxide and cyclic GMP modulate photoreceptor cell responses in the visual system of the locust. J Exp Biol 202:13-20

Seidel C, Bicker G (2002) Developmental expression of nitric oxide/ cyclic GMP signalling pathways in the brain of the embryonic grasshopper. Dev Brain Res 138:71-79

Seki Y, Aonuma H, Kanzaki R (2005) Pheromone processing center in the protocerebrum of Bombyx mori revealed by nitric oxide-induced anti-cGMP immunocytochemistry. J Comp Neurol 481:340-351

Strauss R (2002) The central complex and the genetic dissection of locomotor behaviour. Curr Opin Neurobiol 12:633-638

Stumpner A (1998) Picrotoxin eliminates frequency selectivity of an auditory interneuron in a bushcricket. J Neurophysiol 79:24082415

Trimmer BA, Aprille J, Modica-Napolitano J (2004) Nitric oxide signaling: insect brains and photocytes. Biochem Soc Symp 71:65-83

Vitzthum H, Müller M, Homberg U (2002) Neurons of the central complex of the locust Schistocerca gregaria are sensitive to polarized light. J Neurosci 22:1114-1125

von Helversen O, Elsner N (1977) The stridulatory movements of acridid grasshoppers recorded with an optoelectronic device. J Comp Physiol A 122:53-62

von Helversen D, von Helversen O (1997) Recognition of sex in the acoustic communication of the grasshopper Chorthippus biguttulus (Orthoptera, Acrididae). J Comp Physiol A 180:373-386

Wenzel B, Kunst M, Günther C, Ganter GK, Lakes-Harlan R, Elsner N, Heinrich R (2005) Nitric oxide/cyclic guanosine monophosphate signaling in the central complex of the grasshopper brain inhibits singing behavior. J Comp Neurol 488:129-139

Wessnitzer J, Webb B (2006) Multimodal sensory integration in insects-towards insect brain control architectures. Bioinspir Biomim 1:63-75 
Wicher D, Messutat S, Lavialle C, Lapied B (2004) A new regulation of non-capacitative calcium entry in insect pacemaker neurosecretory neurons. J Biol Chem 279:50410-50419

Wildemann B, Bicker G (1999) Nitric oxide and cyclic GMP induce vesicle release at Drosophila neuromuscular junction. J Neurobiol 39:337-346

Wingrove JA, O'Farrell PH (1999) Nitric oxide contributes to behavioral, cellular, and developmental responses to low oxygen in Drosophila. Cell 98:105-114
Zayas R, Trimmer BA (2007) Characterization of NO/cGMP-mediated responses in identified motoneurons. Cell Mol Neurobiol 27:191209

Zayas R, Qazi S, Morton DB, Trimmer BA (2000) Neurons involved in nitric oxide-mediated cGMP signaling in the tobacco hornworm, Manduca sexta. J Comp Neurol 419:422-438 